\title{
La educación y la investigación ante el COVID-19: Aprendizaje para todos a lo largo de toda la vida
}

\section{Información de artículo:}

Recibido: 15.11 .2020

Aprobado: 30.11 .2020

Palabras claves:

Educación, Investigación, COVID-19, Aprendizaje, Innovación.
William Oswaldo Flores López ${ }^{1}$

\section{Keywords:}

Education, Education, Research, COVID-19, Learning, Innovation.

\section{Resumen}

El COVID-19 ha ralentizado los procesos educativos e investigativos de las Instituciones de Educación Superior. La pandemia, afecta con mayor ímpetu a grupos vulnerables y en riesgo de exclusión social, exacerbando las desigualdades e inequidades sociales entre los distintos sectores de la población. Entonces, es necesario diversificar la educación virtual o digital, mediante un enfoque sistémico del currículo, además, es necesario posicionar un paradigma de investigación descolonizador del pensamiento. En definitiva, la educación y la investigación ante el COVID-19, tratan de promover aprendizaje para todos, a lo largo de toda la vida, mediante procesos propios de construcción del conocimiento que contribuyen a la transformación e innovación de la educación disruptiva.

\section{Education and Research on COVID-19: Lifelong Learning for All}

\begin{abstract}
COVID-19 has slowed down the educational and investigative processes of Higher Education Institutions. The pandemic, affects vulnerable groups and at risk of social exclusion with greater force, exacerbating social inequalities between different sectors of the population. It is necessary to diversify virtual education through a systemic approach to the curriculum, in addition, it is necessary to position a research paradigm decolonizing thought. In short, education and research in the face of COVID-19, try to promote learning for all throughout

\footnotetext{
1 Doctor en Educación. Profesor Investigador de la Universidad de las Regiones Autónomas de la Costa Caribe Nicaragüense. Email: william.flores@uraccan.edu.ni (D): https://orcid.org/oooo-ooo2-1016-1620
} 
life processes of knowledge construction that contribute to the transformation and innovation of disruptive education.

\section{Justificación del monográfico}

El COVID-19 ha ralentizado los procesos educativos e investigativos de las Instituciones de Educación Superior. La pandemia afecta con mayor ímpetu a grupos vulnerables y en riesgo de exclusión social, exacerbando las desigualdades e inequidades sociales entre los distintos sectores de la población, por ejemplo, aunque la educación superior se ha virtualizado no todos los estudiantes procedentes de pueblos indígenas y afrodescendientes tienen acceso a los servicios de internet para interconectarse con las diferentes plataformas educativas.

Investigaciones recientes muestran que esta abrupta transición de la educación presencial a la virtual ha puesto de manifiesto las desigualdades e inequidades que la sociedad en general venía evidenciando en los últimos años (Quiroz-Reyes, 2020). Es evidente que la desigualdad social condiciona cualquier proceso de virtualización de la educación, a no ser que se respalde sobre fuerte inversiones de parte de las instituciones de educación superior que garantice que los insumos, recursos y oportunidades de aprendizaje durante toda la vida para toda la comunidad educativa por igual (Almazán-Gómez, 2020).

En este sentido, es necesario diversificar la educación virtual o digital, mediante un enfoque sistémico del currículo, es decir, los contenidos digitales deberían ir de la mano de otros tipos de medios, tales como la televisión, la radio comunitaria y los medios sociales para llegar a todos los estudiantes, incluyendo aquellos que no cuentan con las tecnologías de aprendizaje, ni la conectividad (UNESCO, 2020). Esto permitiría el cumplimiento de la equidad y la inclusión, que son principios orientadores del objetivo de desarrollo sostenible 4, asumiéndose que ninguna meta educativa debería considerarse lograda a menos que se haya logrado para todos, para lo cual los esfuerzos en las políticas de educación deben centrarse en los grupos más desfavorecidos (UNESCO, 2016, p. 7).

Además, la Conferencia Regional de Educación Superior manifestó que el desafío para la progresiva transformación de las universidades de América Latina y el Caribe se complementa desde los centros de investigación destacados, y advierte, que es necesario recuperar, revalorizar y proteger los conocimientos tradicionales y ancestrales en el marco del respeto a la diversidad, la equidad epistémica y el diálogo de saberes (CRES, 2018). Sin embargo, la actual coyuntura del COVID-19 ha inaugurado una posibilidad histórica para replantear los procesos de investigación (Luna-Nemecio, 2020), mediante el posicionamiento de un paradigma de investigación descolonizador del pensamiento, teniendo de referencia que un paradigma es un sistema de creencias, principios, valores y compromisos grupales que determinan la visión que 
una determinada comunidad tiene de realidad para crear y recrear conocimientos propios de los pueblos.

En este escenario surge el cultivo y crianza de sabidurías y conocimiento como una propuesta pedagógica y metodológica con posicionamiento epistémico y filosófico para el desarrollo de los procesos de creación, recreación de conocimientos, saberes y prácticas de los pueblos, las organizaciones e instituciones. Aporta a la construcción de procesos investigativos de una manera holística, donde las comunidades sean sujetos y no objetos de estudio (RUIICAY, 2018). En definitiva, la educación y la investigación ante el COVID-19, tratan de promover aprendizaje para todos a lo largo de toda la vida mediante procesos propios de construcción del conocimiento que contribuyen a la transformación e innovación de la educación disruptiva.

\section{Estructura y presentación del monográfico}

La Revista Electrónica de Conocimientos, Saberes y Prácticas avanza en los procesos educativos e investigativos a pesar de la crisis mundial del COVID-19, presentando los trabajos siguientes:

En la investigación de Vanegas-Sevilla, Bermúdez-Vargas y Flores (2020) se analizan desde un enfoque ontológico y semiótico un proceso de instrucción en las aplicaciones de la derivada, los autores, lograron evidenciar que los estudiantes tienen actitudes positivas hacia las matemáticas, sin embargo, presentan obstáculos cognitivos básicos para la compresión y resolución de los problemas aplicando las derivadas. De igual manera se evidenció a través del estadístico no paramétrico que existe estadísticamente diferencia entre las medianas entre las pruebas, por tanto, el proceso de instrucción mejora la comprensión y rendimiento académico del estudiantado.

Williams-Muller et al. (2020) proponen la interrogante sobre: ¿Debe incluirse los móviles en las aulas de educación primaria?, los autores reconocen inconvenientes sobre su uso, las cuales están relacionado con la distracción, dependencia y la desigualdad que genera en el aula de clase. Pero, se reconoce la necesidad de su incorporación, esta debe ir función de la formación del profesorado, capacitación del estudiantado y padres de familia.

El estudio de Flores-Morales y Mondragón-Marín (2020), aborda el aporte socioeconómico de la empresa aceitera San José, ofrece en la Región Autónoma de la Costa Caribe Sur de Nicaragua, el autor revela diversos programas sociales hacia la comunidad y de impacto positivo hacia los colaboradores, concretizando sus políticas de premiación a su labor en la empresa. Además, la empresa brinda apoyo humano, técnicos o financieros para ayudar a los colectivos menos favorecidos en la sociedad. Existe una política interna de apoyo a los colaboradores, y éstas se encuentran dentro de los documentos oficiales de la Empresa Aceitera, pero se requiere que la empresa 
dé a conocer a sus colaboradores los aportes que realiza en materia social y económica en el Rama, para ello debe destinar tiempo en reuniones $u$ asambleas generales para informar del quehacer de la empresa.

En la investigación de Sandoval (2020) se indaga sobre las estrategias para la enseñanza y el aprendizaje del español como lengua materna. Se trata de un análisis de 45 trabajos didácticos de estudiantes universitarios de Lengua y Literatura Hispánica de la Universidad Nacional Autónoma de Nicaragua, Managua, divididos entre las diferentes macrohabilidades: escritura, expresión oral, comprensión lectora, gramática y literatura. Los principales resultados manifiestan que la utilización del modelo de aprendizaje por esquema facilita la reestructuración y la generación de una nueva secuencia didáctica de los trabajos de los estudiantes. Se concluye, que el modelo de aprendizaje por esquema fortalece la competencia didáctica y de investigación de los estudiantes.

En Gutiérrez-Mendoza (2020a) se propone una reflexión sobre los sistemas de pensamientos propios de pueblos indígenas y afrodescendientes vigentes en la actualidad, frecuentemente invisibilizados por la ausencia del rigor científico. Desde la experiencia de la universidad comunitaria intercultural se realizan planteamientos de construcción colectiva; de creación, recreación y prácticas de saberes y conocimientos, de los pueblos. A largo de toda la discusión se presentan reflexiones y replanteamientos para actuar en la Educación Superior, escenario de instauración de sociedades del conocimiento. Finalizando con afirmaciones y convicciones de que la educación superior intercultural debe ser para la conservación de las culturas; valoración de la diversidad; y legitimar los sistemas de pensamientos propios.

En la investigación de Díaz-Pérez y Cruz-Acevedo (2020) se expone que en el siglo XXI ha sido un siglo de cambios educativos sustanciales, el profesorado se ha visto envuelto en un proceso profundo de autorreflexión pedagógica que lo ha conllevado a la apropiación del discurso de las innovaciones educativas como un elemento indispensable para obtener mejoras en los resultados educativos. Entre este entramado de innovaciones puestas en el centro de discusión del debate académico, el presente ensayo se plantea como finalidad generar un espacio de reflexión educativa entre profesores en ejercicio y en formación, entorno a las metodologías participativas de los estudiantes en las clases de ciencias sociales como factor esencial dentro de la innovación pedagógica. Para ello, el ensayo inicia haciendo un denso recorrido por las problemáticas relacionadas con las metodologías didácticas y el poco protagonismo que tiene el estudiante en las mismas, luego plantea el tema de las metodologías participativas como un elemento de innovación educativa en los procesos de aprendizajes, para así finalmente sentar las bases de los retos que tiene el profesorado respecto a la implementación de metodologías participativas en el aula de clase, tomando como referencia principal el contexto de la sociedad que avanza e innova día a día. 


\section{EDITORIAL}

En Hernández-Castellón y Zamora-Díaz (2020) se reflexiona sobre los sistemas de gestión de la calidad de las Instituciones de Educación Superior Nicaragüense. Se trata de una revisión bibliográfica que interioriza, reflexiona y genera aportes sobre calidad y gestión educativa. En este sentido, se aprecia una relación directa entre una buena gestión universitaria desde las distintas áreas y la calidad del servicio educativo; y la aplicación de normas de calidad es una herramienta de gestión que promueve la competitividad y garantiza la excelencia académica. En definitiva, se evidencia la necesidad de implementar sistemas de gestión que permitan alcanzar altos niveles de desempeño organizacional y al mismo tiempo, excelentes resultados de aprendizaje por parte de los estudiantes.

Finalmente, Gutiérrez-Mendoza (2020b) reflexiona que los pueblos indígenas y afrodescendientes en las luchas de reinvidicación de derechos han desarrollado la capacidad de liderazgo, para impulsar y movilizar las acciones en función de las aspiraciones propias, que conduzcan a la vida en plenitud, Buen Vivir. Entorno a las conceptualizaciones de liderazgo, este referente plantea una estrecha relación entre liderazgo educativo y liderazgo comunitario, partiendo de la influencia que tienen los mismos para trazar los caminos del Buen Vivir de los pueblos indígenas y afrodescendientes desde los procesos Autonomía y Educación. Se aborda el tema de liderazgo educativo,desde la experiencia del Sistema Educativo Autonómico Regional como modelo de Educación propia que surge de la colaboración conjunta; igualmente se describe los puntos de partida de las entidades de educación superior, liderazgos educativos de acción formativa en ideales filosóficos propios para el bien común y en convivencia armónica. En la última sección se puntualizan los desafíos que tienen las instituciones educativas para realizar liderazgo educativo en materia de educación intercultural.

\section{Lista de referencias}

Almazán-Gómez, A. (2020). Covid-19: ¿Punto sin retorno de la digitalización de la educación? Revista Internacional de Educación para la Justicia Social (RIEJS).

Díaz-Pérez, A. A., \& Cruz-Acevedo, A. A. (2020). Hacia un modelo de clases participativas de Ciencias Sociales en Educación Secundaria. Revista Electrónica de Conocimientos, Saberes y Prácticas, 3(2), 79-86. https://doi.org/10.5377/recsp. v3i2.10693

Flores-Morales, J., \& Mondragón-Marín, P. (2020). Aporte socioeconómico de la empresa aceitera San José en El Rama, Región Autónoma de la Costa Caribe Sur de Nicaragua. Revista Electrónica de Conocimientos, Saberes y Prácticas, 3(2), 43-53. https://doi.org/10.5377/recsp.v3i2.1069o. 
Gutiérrez-Mendoza, S. Y. (2020a). Sistemas de pensamientos propios: Un aprendizaje desde la Universidad Comunitaria Intercultural. Revista Electrónica de Conocimientos, Saberes y Prácticas, 3(2), 66-78. https://doi.org/10.5377/recsp. v3i2.10692

Gutiérrez-Mendoza, S. Y. (2020b). Un modelo de liderazgo y educación propia para el Buen Vivir y la Autonomía de los pueblos. Revista Electrónica de Conocimientos, Saberes y Prácticas, 3(2), 98-109. https://doi.org/10.5377/recsp.v3i2.10695

Hernández-Castellón, J. A., \& Zamora-Díaz, W. J. (2020). Sistemas de gestión de la calidad: Una mejora en la calidad de las Instituciones de Educación Superior en Nicaragua. Revista Electrónica de Conocimientos, Saberes y Prácticas, 3(2), 87-97. https://doi.org/10.5377/recsp.v3i2.10694

Luna-Nemecio, J. (2020). Ciencias Sociales y COVID-19: retos, vicisitudes y oportunidades para la investigación. FORHUM International Journal of Social Sciences and Humanities, 2(3), 6-12.

Quiroz-Reyes, C. (2020). Pandemia Covid-19 e Inequidad Territorial: El Agravamiento de las Desigualdades Educativas en Chile. Revista Internacional de Educación para la Justicia Social, 9(3).

Sandoval, A. J. (2020). Competencia didáctica en estudiantes universitarios de Lengua y Literatura Hispánicas. Revista Electrónica de Conocimientos, Saberes y Prácticas, 3(2), 54-65. https://doi.org/10.5377/recsp.v3i2.10691

UNESCO. (2016). Educación 2030: Declaración de Incheon y Marco de Acción para la realización del Objetivo de Desarrollo Sostenible 4, París.

UNESCO. (2020). Respuestas del ámbito educativo de la UNESCO al COVID-19. Notas temáticas del sector educativo, 42. https://es.unesco.org/covid19/educationresponse

Vanegas-Sevilla, E. E., Bermúdez-Vargas, Y., \& Flores, W. O. (2020). Análisis ontosemiótico del proceso de instrucción en aplicaciones de la derivada con el estudiantado de Ingeniería Agroforestal. Revista Electrónica de Conocimientos, Saberes y Prácticas, 3(2), 10-30. https://doi.org/10.5377/recsp.v3i2.10686

Williams-Muller, E., Castro-Galviz, M. Y., De la Cruz-López, M., Nogales-Pinillas, L., Roldán-García, L., Torres-Carvalho, J. L. (2020). Uso de los teléfonos móviles en el aula de educación primaria. Revista Electrónica de Conocimientos, Saberes y Prácticas, 3(2), 31-42. https://doi.org/10.5377/recsp.v3i2.10688 